\title{
Bilinguismo, educazione linguistica e interculturalità: il caso delle sezioni bilingui italo-albanesi a Scutari, Albania
}

\author{
Lindita Kazazi \\ Università di Scutari, Albania \\ lindita.kazazi@unishk.edu.al \\ Eliana Laçej \\ Università di Scutari, Albania \\ eliana.lacej@unishk.edu.al
}

I contesti sociali, di cui siamo partecipi tutti noi, si possono individuare come società segnate sempre di più dal multiculturalismo e dal multilinguismo. La diversità e la pluralità linguistica, il legame tra la lingua materna e le altre lingue, le implicazioni tra questa dimensione dello sviluppo e le altre si segnano come temi poco declinati dal punto di vista pedagogico ed educativo. Infatti, l'educazione plurilingue e interculturale risponde al diritto di ogni individuo ad una educazione di qualità: acquisizione di competenze, di conoscenze, di strategie e di atteggiamenti, diversità delle esperienze di apprendimento, costruzione di identità culturali individuali e collettive. Si tratta di rendere più efficace l'insegnamento e allo stesso tempo di far sì che contribuisca in modo maggiore al successo nell'educazione linguistica vista come integrazione tra lingua materna e lingua/e straniera/e. Il fenomeno dell'esistenza di contesti linguistici in cui la competenza comunicativa nella lingua straniera (concretamente, l'italiano) è aggiunta perfettamente alla competenza comunicativa nella lingua madre (qui, l'albanese) costituisce un caso particolare di bilinguismo. Tale fenomeno, riscontrato non solo in contesti guidati, ma anche e soprattutto in contesti misti, suscita a studiare i motivi storici, geografici e sociali, a valorizzare il plurilinguismo e a stimolare la riflessione linguistica a partire dal confronto tra L1, L 2 e altre lingue straniere per arrivare il meglio possibile ad un'educazione linguistica e interculturale verso un mondo sempre più globalizzato.

Parole chiave: bilinguismo, didattica, competenza interculturale (c) BY-SA https://doi.org/10.26493/978-961-293-045-5.51-61

\section{Introduzione}

In ogni lingua esiste un lessico stabile negli usi, spesso antichissimo, ed un altro fluttuante, corrispondente ai bisogni di un determinato periodo storico. Nel caso della lingua albanese, le parole della prima categoria ap- 
partengono in gran parte all'antico fondo indoeuropeo o ai prestiti latini, slavi, turchi, greci e italiani. La precisazione fornita agli studiosi da questi elementi, la possibilità offerta per stabilirne una cronologia relativa e la loro parte attiva nella lingua albanese contemporanea, fanno dell'influenza latina un mezzo di orientamento con punti di riscontri indispensabili per la storia della lingua albanese. Gli elementi latini che ammontano a 600 (Mihaescu 1979, 26) appaiono in molti ambiti, il che è testimonianza dell'intensità della romanizzazione. L'influenza dell'italiano, a conseguenza di contatti spessissimi e intensi tra il mondo albanese e quello italiano, è pervenuta come in un continuum dell'influsso latino sulla madre dell'albanese. Gli studiosi affermano l'esistenza di duplici prestiti, ossia di parole imprestate dal latino e in seguito dall'italiano (Gjinari e Shkurtaj 2003, 326).

La lingua italiana a Scutari è vista come un vero e proprio territorio linguistico perché la sua presenza è talmente forte da giustificare tale denominazione. Scutari è una di quelle città che forse più di altre in Albania ha risentito la forte presenza dell'Italia e dell'italianità e questo non solo a causa della vicinanza geografica ma anche per ragioni storiche, politiche e sociali e culturali che hanno caratterizzato fitti rapporti di interscambi nei secoli.

Per capire la forte presenza della lingua italiana a Scutari bisognerebbe indagare nelle radici remote della storia che ha legato per secoli i due popoli. Cominciando dai rapporti commerciali già dai tempi dei romani, con gli scambi navali avvenuti nel porto del Lago di Scutari che un tempo ospitava il più grande mercato dei Balcani, per continuare poi nel medioevo con i rapporti politici e commerciali con Venezia, le cui tracce si riconoscono nella lingua albanese in generale e scutarina in particolare. ${ }^{1}$

Importantissimi sono anche i legami religiosi. Da ricordare a questo proposito la presenza dei Francescani che risale alla prima metà del XVI secolo. Tramite gli ordini religiosi si fondavano opere in cui si utilizzava la lingua della nazione di provenienza del clero quindi durante quel periodo conventi, seminari, scuole, asili, collegi, ospedali, orfanotrof, ospizi, praticavano l'italiano come lingua veicolare, accanto ovviamente all'albanese e ad altre lingue. Non è un caso quindi che la prima scuola albanese di tipo religioso sia stato un seminario francescano fondato nel 1861 a Scutari, anche se ci sono citazioni di scuole albanesi dei Francescani dal 1638 in Pdhanë (Dema 1940, 606).

\footnotetext{
${ }^{1}$ Sugli influssi del veneziano nella lingua albanese Vidos (1962), Landi (1989) e Vicario (1999).
} 
Nei secoli, nonostante i vari domini su Scutari, i rapporti con l'Italia non sono stati interrotti ufficialmente fino all'avvento del regime comunista il quale chiude i confini e proibisce ogni tipo di contatto con la penisola. Tuttavia, anche durante questo periodo la presenza italiana rimane attiva tramite le trasmissioni televisive e radiofoniche che venivano viste o ascoltate clandestinamente, rischiando anche il carcere. Lo stesso studio della lingua italiana avveniva in modo clandestino. Da fonti orali veniamo a sapere che i corsi di lingua italiana venivano svolti anche nei parchi della città, nei quali facendo le passeggiate si praticava l'italiano migliorando in questo modo l'abilità del parlato. La caduta del regime comunista, nel 1990, segnò anche la ripresa dei contatti persi per arrivare ad oggi quando l'Italia rappresenta per l'Albania il primo partner commerciale e gli Albanesi in Italia oggi costituiscono una comunità straniera ben integrata.

La storia di intensi scambi economici, storici e culturali e sociali è quindi alla base delle ragioni per cui, per secoli, gli scutarini, come d'altronde tutti gli albanesi, si sono avvicinati allo studio della lingua italiana.

È da sottolineare in aggiunta l'importanza dei mass media nella diffusione della lingua italiana. Se durante il periodo pre-comunista l'italiano era una lingua veicolare che veniva studiata a livello scolastico e appresa attraverso contatti con parlanti nativi, durante il periodo comunista, una delle grandi fonti della diffusione della lingua italiana in Albania, oseremmo dire la maggiore, è stata la televisione. La televisione è stato il grande mezzo che ha veicolato la lingua italiana in Albania, e in particolar modo a Scutari, la quale vantava la vicinanza con il Montenegro e quindi aveva maggiore possibilità di captare le onde radiotelevisive italiane. Gli albanesi che hanno appreso l'italiano tramite la $\mathrm{TV}$ possiedono delle conoscenze dell'italiano più o meno standard, sicuramente influenzate a livello fonetico dalla loro L1.

Negli anni 1990-2005 la televisione italiana ha avuto in Albania una posizione quasi di monopolio. In questo quindicennio, la televisione italiana, grazie ai suoi numerosi canali e all'estrema varietà di trasmissioni, e continuando a essere ancora la televisione preferita, ha colmato e sta ancora colmando il vuoto di quella albanese.

L'acquisizione della lingua italiana tramite la televisione, a partire dal 1990, quindi è stata frutto essenzialmente di fatti puramente tecnici, commerciali e linguistici: tecnici perché le onde radiotelevisive non incontravano barriere; commerciali perché la televisione italiana piaceva per la varietà dei suoi programmi; linguistici perché la lingua italiana era intrinsecamente più intuibile e intelligibile di tante altre lingue straniere. Tutto questo 
ha favorito la diffusione dell'italofonìa, insperabile con altri mezzi e in un periodo così breve.

Oggigiorno ci troviamo di fronte ad una situazione alquanto cambiata. La diffusione della lingua italiana tramite la TV dal 2005 è in calo. L'italiano ormai non è una lingua la cui padronanza si dà per scontata. Di anno in anno essa deve competere con l'inglese, il francese o anche lo spagnolo o portoghese, lingue che stanno occupando il terreno dell'italiano, soprattutto a livello televisivo. Ultimamente sono molto seguiti ed amati anche $\mathrm{i}$ telefilm in lingua turca. Considerando che gli albanesi guardano molto la TV imparando le lingue tramite essa, si può intuire che le lingue nominate sopra stiano pian piano occupando parte del terreno linguistico dell'italiano. Se prima gli studenti si presentavano a scuola con un considerevole bagaglio linguistico e culturale italiano, ora possiedono una formazione linguistica e culturale inglese o spagnola. In particolar modo quest'ultimo sta disturbando molto l'italiano con il suo continuo intercalare. Gli studenti guardano le telenovelas in spagnolo e a causa delle somiglianze tra le due lingue tendono a confonderle. In definitiva risulta quindi che la conoscenza della lingua italiana di base non è più scontata come una volta, dunque c'è bisogno di interventi mirati da parte dei responsabili delle politiche scolastiche. Attualmente nelle scuole pubbliche e private di Scutari, come nel resto nelle scuole dell'intera Albania, la lingua italiana è presente nelle scuole dell'obbligo, nelle scuole superiori e all'università. Essa viene studiata come prima o seconda lingua straniera.

Nelle scuole dell'obbligo, pubbliche ed in alcune private, lo studio della lingua italiana inizia dalle medie inferiori (v/vi classe) come seconda lingua straniera. Fanno eccezione le scuole del progetto Illiria e della Sezione Bilingue, dove l'insegnamento dell'italiano, considerato come prima lingua straniera, inizia dalla II I e V I classe e si svolge rispettivamente per 3 e 5 ore a settimana. Fanno eccezione anche alcune scuole private religiose, come la scuola «Cor Jesu» in cui l'insegnamento inizia già dalla I classe elementare. Il processo formativo continua fino alla IX classe.

Nelle scuole secondarie superiori, dalla X classe alla XI I, l'insegnamento della lingua italiana continua secondo i seguenti percorsi di studio:

1. Gli studenti che hanno iniziato lo studio della lingua dalla II I classe, quindi come prima lingua straniera, dovrebbero continuare a studiarla come tale, con 3 ore settimanali in X e XI classe e 4 ore settimanali in XI I classe. Il livello linguistico in uscita di questi studenti dovrebbe essere B2+. 
2. Gli studenti che hanno iniziato lo studio della lingua dalla vi classe, quindi come seconda lingua straniera, hanno la possibilità di continuare a studiarla in $\mathrm{X}$ come tale. All'inizio dell'anno scolastico, essi dovrebbero selezionare l'italiano come materia da svolgere tra le altre discipline della categoria a scelta obbligatoria. Il processo didattico si svolge con 2 ore settimanali ed il livello linguistico in uscita dovrebbe essere un B2.

3. Gli studenti che non hanno imparato la lingua italiana nella scuola media inferiore hanno la possibilità di iniziare in X. Anche per questi studenti l'italiano viene considerato come seconda lingua straniera. Il processo didattico si svolge con 2 ore settimanali ed il livello linguistico in uscita dalla classe XII è B1.

A livello universitario, l'italiano è presente presso l'Università di Scutari «Luigj Gurakuqi» già dagli anni'9o, dopo la caduta del comunismo, nei corsi di italiano come lingua straniera insegnata, nei corsi di laurea nelle varie facoltà e dal 1998 nel corso di laurea in lingua italiana che è istituito presso la Sezione di Italianistica del Dipartimento di Romanistica, della Facoltà di Lingue Straniere.

Fino al 2005 il percorso di studio è stato quadriennale, da quell'anno in poi è stato adottato anche in Albania il modello $3+2$ (1) anni del «Processo di Bologna» che suddivide il corso di studi in due segmenti, con laurea di I livello, seguita da quella del Master con lo sbocco professionale «Insegnante di lingua italiana». Sono attualmente in corso di preparazione altri corsi del Master con specializzazione in Traduzione, Interpretariato, Turismo e Patrimonio culturale, percorsi che aprono, in tal modo, vari sbocchi professionali.

Inoltre, sempre all'Università, la lingua italiana è offerta anche come lingua straniera. Nell'anno accademico 2019/2020 la lingua italiana viene studiata come LS da circa 250 studenti. L'italiano si trova a competere nello stadio attuale oltre che con l'inglese anche con il tedesco, una lingua molto richiesta negli ultimi 3-5 anni.

L'Università di Scutari si è avvalsa di un'intensa collaborazione con l'Università di Firenze per circa 10 anni. A riguardo, evidenziamo scambi di docenti e ricerche nell'applicazione delle tecnologie informatiche e dei linguaggi multimediali alla didattica. La Sezione d'Italianistica dell'Università di Scutari è sostenuta dal Governo italiano con l'assegnazione di un professore di ruolo italiano con funzione di Lettore, oltre che con rilevanti contributi per il pagamento di lettori locali e per il loro aggiornamento di- 
dattico, per progetti didattici speciali e per la fornitura di libri e materiali didattici. Gli investimenti del governo italiano sono estesi anche agli altri livelli scolastici ma di ciò parleremo più avanti.

Il clero continua a sostenere l'insegnamento della lingua italiana nelle scuole private religiose in cui opera ed a Scutari se ne contano molte. Alcune di queste sono: «Sacro Cuore» - Scuola dell'obbligo/Scuola materna - Suore Missionarie del Sacro Costato; «Cor Jesu» - Scuola dell'obbligo/Scuola materna - Suore Apostole del Sacro Cuore di Gesù; QAFS «Maria Ausiliatrice» - Liceo/Scuola dell'obbligo/Scuola materna; «At Pjeter Meshkalla» - Liceo - Compagnia di Gesù ecc.

Alle iniziative di queste istituzioni si aggiungono anche quelle dei vari centri di formazione professionali o anche di alcuni centri linguistici privati che organizzano corsi di lingua e, inoltre, le scuole religiose non cattoliche che propongono corsi di lingua italiana.

Per sostenere e rinforzare lo studio della lingua italiana in Albania il Governo italiano, in collaborazione con quello albanese, ha avviato una serie di programmi per l'insegnamento della lingua italiana. Presentiamo qui una breve lista di programmi e sedi di studio della lingua italiana a Scutari.

\section{Il programma Illiria}

Si tratta di una convenzione politica siglata da un accordo bilaterale tra il Governo italiano e quello albanese che regolamenta l'insegnamento dell'italiano come prima lingua straniera nelle scuole dell'obbligo e quelle superiori, avendo come obiettivo l'avvicinamento alla lingua e cultura italiana e fornendo un contatto formativo di notevole importanza per la crescita culturale la cui spendibilità è rilevante se si considerano gli attuali rapporti bilaterali tra Italia e Albania e, soprattutto, le prospettive di sviluppo degli interscambi culturali e commerciali tra i due Paesi.

Questo importantissimo programma è stato avviato nel 2002 nelle terze classi delle scuole dell'obbligo e nelle prime classi delle scuole secondarie superiori, con lo scopo di aumentare il numero di studenti ai quali consentire successivamente la scelta dell'italiano come prima lingua straniera in tutte le scuole in cui si prevede lo studio di una o più lingue straniere. Nelle ore di insegnamento c'è la compresenza di un insegnante albanese e uno italiano. La lingua di comunicazione in classe è l'italiano, anche se a volte i ragazzi possono presentare alcune difficoltà di comprensione. È da sottolineare l'uso esclusivo dell'italiano anche da parte degli insegnanti albanesi che non avviene in altre sedi del programma nelle altre città albanesi. Questo fatto è testimoniato dagli insegnanti stessi che riconoscono in 
queste sezioni un'oasi bilingue, nelle quali l'italiano può essere considerato più una L 2 che una LS.

A Scutari, il programma è esteso nella scuola di base «Ndre Mjeda» e fino all'anno scolastico 2010/2011 anche alla scuola secondaria «Oso $\mathrm{Ku}$ ka». Il governo italiano sostiene queste scuole con materiale didattico e contribuisce alla formazione dei docenti.

\section{La sezione bilingue italo-albanese}

Costituendo un piccolo territorio italiano nelle scuole albanesi, il via alle sezioni bilingui italo-albanesi è stato dato il 26 aprile 2002 attraverso la firma del Memorandum d'Intesa tra i due governi. Nell'ottica bilingue e biculturale, l'obiettivo delle sezioni bilingui è l'insegnamento della lingua e cultura italiana a parità con la lingua madre albanese e l'utilizzo veicolare dell'italiano per altre discipline umanistiche e scientifiche. Nel 2011 è stato firmato un nuovo Memorandum d'Intesa che prevede lo studio della lingua italiana già nelle medie inferiori (V I-IX classe) per continuare poi nelle scuole medie superiori o Gjimnaz (X-X I I classe). ${ }^{2}$ Al liceo è previsto lo studio della lingua e letteratura italiana e della matematica per 5 ore, nonché delle altre discipline in lingua italiana (Fisica, Biologia, Storia universale e Storia dell'arte) per più del 50\% delle ore settimanali, secondo lo schema, presentato nella tabella 1.

Il percorso formativo delle Sezioni Bilingui italo-albanesi è dunque umanistico-scientifico e la finalità è una formazione d'eccellenza che garantisca agli studenti un insieme di conoscenze e competenze spendibili tanto in Italia quanto in Albania, sia per il proseguimento degli studi superiori sia sul mercato del lavoro, oltre alla capacità di essere i facilitatori nelle relazioni tra i due Paesi. Il processo didattico in queste sezioni si attua in compresenza di insegnanti italiani ed albanesi in un clima di collaborazione e scambio di metodologie e di esperienze lavorative. Materie quali letteratura, storia, fisica e matematica si svolgono con la compresenza di entrambi gli insegnanti. La materia di storia dell'arte si svolge solo con insegnante italiano, il che favorisce gli studenti che desiderano studiare architettura in Italia, fornendo loro informazioni specifiche dell'ambito, nonché' un'eccellente formazione linguistica del linguaggio del settore.

${ }^{2}$ L'insegnamento dell'italiano nel sistema scolatico pubblico albanese pubblicato dall'Ufficio della Cooperazione Scolastica sul sito dell'Ambasciata Italiana a Tirana, si veda il sito dell'Ambasciata Italiana a Tirana (https://ambtirana.esteri.it/ambasciata_tirana/it/i_rapporti _bilaterali/cooperazione\%2oculturale/ufficio-scuole_o.html). 
Tabella 1 Piano di studi e ore di insegnamento nella sezione bilingue

\begin{tabular}{|c|c|c|c|c|}
\hline \multirow[t]{2}{*}{ Discipline } & & \multicolumn{3}{|c|}{ Classe } \\
\hline & & $\mathrm{x}$ & XI & XII \\
\hline \multirow[t]{7}{*}{ Discipline in italiano } & Lingua e letteratura italiana & 5 & 5 & 5 \\
\hline & Storia universale & 2 & 2 & 2 \\
\hline & Matematica & 5 & 5 & 5 \\
\hline & Fisica & 3 & 3 & 3 \\
\hline & Biologia & 2 & 2 & 2 \\
\hline & Storia dell'arte & 1 & 1 & 1 \\
\hline & Totale ore in italiano & 18 & 18 & 18 \\
\hline \multirow[t]{11}{*}{ Discipline in albanese } & Lingua e letteratura albanese & 3 & 3 & 4 \\
\hline & Storia albanese & $\mathrm{o}$ & $\mathrm{o}$ & 2 \\
\hline & Lingua inglese & 3 & 3 & 4 \\
\hline & Economia & o & o & 2 \\
\hline & TIC & 2 & 1 & $\mathrm{o}$ \\
\hline & Chimica & 2 & 2 & o \\
\hline & Filosofia & o & o & 1 \\
\hline & Sociologia & & 1 & \\
\hline & Geografia & $\mathrm{o}$ & 1 & 2 \\
\hline & Educazione fisica & 2 & 1 & $\mathrm{o}$ \\
\hline & Totale ore in albanese & 12 & 12 & 15 \\
\hline
\end{tabular}

Il grado di conoscenza della lingua italiana in uscita dalla classe X I I è pari al C1. Il diploma di maturità conseguito in queste sezioni è riconosciuto anche in Italia e di conseguenza anche negli altri stati dell'U E. Gli studenti diplomati presso queste sezioni possono iscriversi alle Università italiane, agli stessi corsi ai quali il diploma dà accesso nelle Università albanesi, con esonero dalla prova di lingua italiana e al di fuori del limite dei posti normalmente previsti per gli studenti stranieri. I migliori alunni, iscritti presso le Università italiane, ricevono borse di studio del Ministero Affari Esteri Italiano. A Scutari la Sezione Bilingue ha sede presso le scuole medie «Ndre Mjeda», «Ismail Qemali» e presso il Liceo linguistico «Shejnase Juka» ed il Liceo «28 Nentori».

\section{Corsi di lingua italiana}

Rilevante importanza nell'ambito dell'insegnamento della lingua italiana ha la Società Dante Alighieri presente a Scutari. Presso la Sezione di Italianistica dell'Università si possono frequentare corsi di lingua italiana per 
ottenere la certificazione P L I D A ${ }^{3}$ (Progetto Lingua Italiana Dante Alighieri) la quale attesta la competenza in lingua italiana come lingua straniera. P L ID A sta ultimamente organizzando dei corsi di lingua anche per bambini e adolescenti con l'intenzione di incentivare la passione verso la lingua e la cultura italiana.

L'Istituto italiano di Cultura è presente a Scutari con corsi di lingua italiana a cura dei docenti locali ed articolati su tre livelli. I corsi, normali o intensivi, sono organizzati a seconda del livello di conoscenza posseduto. Ogni anno gli iscritti ai corsi hanno l'opportunità di sostenere l'esame del CE LI (Certificato di Lingua Italiana a livello 2, 3, 4, 5) organizzato dall'Università per Stranieri di Perugia.

Come si può facilmente intuire l'offerta didattica per lo studio della lingua italiana a Scutari è ampia e varia. Essa è presente nelle istituzioni educative statali e religiose, pubbliche e private e si estende lungo tutto il percorso della formazione scolastica, cominciando dalle classi elementari fino a livello accademico universitario. L'obiettivo centrale non è solo quello del mantenimento della predominanza linguistica italiana ma anche della formazione di base linguistico-culturale per coloro che entreranno a far parte del mercato di lavoro. L'italiano infatti è prevalentemente lingua del lavoro ma anche di relazioni sociali e culturali.

Nonostante la considerevole presenza italiana a Scutari da quella diplomatica e religiosa alle numerose imprese finanziarie, sociali, commerciali ed industriali la triste verità dimostra un calo del numero degli interessati all'apprendimento della lingua italiana.

\section{Conclusioni}

Di anno in anno, le politiche educative del governo albanese stanno privilegiando l'insegnamento di altre lingue, soprattutto dell'inglese che per alcuni anni è stata una lingua obbligatoria ai fini dell'ottenimento di titoli universitari ed accademici, a discapito di altre lingue straniere tra cui anche l'italiano. È quindi il momento di puntare verso nuove strategie che favoriscano la motivazione alla scelta dello studio dell'italiano come lingua

${ }^{3}$ Questo è un diploma ufficiale rilasciato dalla Società Dante Alighieri in base a una convenzione con il Ministero degli Affari Esteri (n. 1903 del 4/11/1993) d'intesa con l'Università «La Sapienza» di Roma (convenzione del 29/6/2004). Il P L I D A è riconosciuto anche dal Ministero del Lavoro e delle Politiche Sociali (decreto 18/10/2002). Inoltre il Ministero dell'Università e della Ricerca riconosce al certificato di competenza linguistica P L I D A la validità di titolo che agevola l'immatricolazione universitaria agli studenti stranieri (convenzione 9/10/2006). 
straniera. Strategie che prevedano percorsi formativi linguistici finalizzati alla formazione di operatori destinati ad un concreto mercato di lavoro e/o agli interscambi economici, sociali e culturali.

L'infrenabile evoluzione tecno-scientifica, inoltre, crea la necessità di revisione e continuo aggiornamento dell'insegnamento con nuove tecniche e metodi che più si avvicinano alle esigenze ed al profilo dello studente di oggi. La nuova frontiera dell'insegnamento delle lingue è l'e-learning e l'implementazione di tale programma si sta già considerando anche per l'italiano.

Una speciale passione culturale anima gli italianisti albanesi e li spinge verso nuove idee e strategie al fine di non perdere terreno, comunque non si può avviare niente se non ci si avvale anche di un forte sostegno politico scolastico ed economico dei due governi. Ci auguriamo che l'amicizia particolare tra Italia ed Albania, come da tradizione, continui a fare dell'italiano la principale lingua straniera che appassiona gli albanesi.

\section{Riferimenti bibliografici}

Dema, B. 1940. «Shtypi franceskan ne Shqypni.» Hylli i drites 16 (12): 605-628. Gjinari, J., e J. Shkurtaj. 2003. Dialektologjia. Tirana: S H B L U.

Landi, A. 1989. «I lessici inversi come strumento di identificazione di elementi alloglotti: italianismi nella lingua albanese contemporanea.» In L'italiano allo specchio: aspetti dell'italianismo recente, a cura di L. Coveri, 93-105. Torino: Rosenberg e Sellier.

Mihaescu, H. 1979. «I prestiti latini nell'albanese». Zjarri, n. 1-2: 49-67.

Vicario, F. 1999. «Note sulla diffusione del lessico veneziano nella penisola balcanica.» In Processi di convergenza e differenziazione nelle lingue dell'Europa medievale e moderna: atti della conferenza internazionale, Udine, 9-11 dicembre, 227-241. Udine: Forum.

Vidos, B. E. 1962. «I problemi dell'espansione della lingua nautica veneziana con particolare riguardo all'Oriente Balcanico.» Bollettino dell'Atlante linguistico mediterraneo, n. 4: 13-20.

\section{Dvojezičnost, jezikovna vzgoja in medkulturnost: primer italijansko-albanskih dvojezičnih oddelkov v Skadru, Albanija}

Predstavitev zgodnjih povezav med jadranskima obalama ni preprosta, saj so te povezave kompleksne in raznolike. Povezovanje med Italijo in Skadrom zaznamujejo edinstvene večplastne dimenzije. $V$ prispevku prikazujemo zgodnje povezave, ki segajo v latinsko obdobje, odnose z Beneško republiko, zgodnje obdobje prejšnjega stoletja, ki ga je zaznamovalo odprtje šol verskega značaja, leta grenke diktatorske izolacije in obdobje po njej v 9o-ih letih. Pri tem predstavljamo predvsem razširjenost italijanskega jezika v Al- 
baniji v različnih obdobjih in zastopanost italijanščine na različnih ravneh izobraževalnega sistema. V Skadru je poučevanje italijanskega jezika potekalo stalno in neprekinjeno, včasih prikrito ( $v$ času diktature), sicer pa odprto. Pomembno sredstvo za širjenje italijanskega jezika je bila nedvomno tudi televizija $\mathrm{z}$ italijanskimi kanali, edino okno, skozi katero so Albanci v času politične izolacije gledali svet. V Skadru je učenje italijanščine intenzivno predvsem $\mathrm{v}$ dvojezičnih oddelkih devetletne osnovne šole in srednje šole, ki so prave dvojezične oaze, kjer se albanski in italijanski jezik stalno prepletata. V zadnjih letih se italijanski jezik sooča z nesporno nadvlado angleškega jezika, ki je trenutno prvi tuji jezik v izobraževanju.

Ključne besede: dvojezičnost, didaktika, medkulturna zmožnost

\section{Bilingualism, Language Education and Interculturality: The Case of the Italian-Albanian Bilingual Sections in Shkoder, Albania}

Presenting the early links between the two Adriatic coasts is not easy, given the complexity and variety of these relationships. In the case of Shkodra's connections with Italy, the multifaceted dimensions are themselves unique. In the paper we tried to testify to the early links, in the Latin period, to relations with the Republic of Venice, the first years of '90o with the opening of schools of religious character, the years of bitter dictatorial isolation and the post-years period in the 1990s. Throughout this journey, we mainly focused on spreading the Italian language in Albania and extending its learning across different education cycles. Undoubtedly the teaching of the Italian language in Shkodra has been continuous and uninterrupted, sometimes in hidden form (during the dictatorship) and in open form in other periods. The means of spreading the Italian language has also been undoubtedly television with Italian channels, the only window through which Albanians viewed the world. Italian in Shkodra is heavily studied in the Bilingual Sections of the Nine-Year and Secondary Cycles. These contexts of learning the Italian language can be considered as bilingual oases, where the Albanian and Italian languages always go hand in hand. In recent years the Italian language has been struggling with the undisputed supremacy of the English language which has now become the first foreign language studied.

Key words: bilingualism, didactics, cross-cultural competence 